\title{
Powering catalysis with supercomputers
}

\author{
Catalysis research has immensely benefited from the use of high-performance computing facilities. On the \\ occasion of the twenty-fifth anniversary of the first Top500 list, we briefly revisit its content and evolution \\ and the impact that supercomputers have had in catalysis.
}

C omputational catalysis is a field that has been progressively growing since its early days, and has experienced a surge particularly in recent decades. The formulation of quantum mechanics and the subsequent development of methods to solve the Schrödinger equation in the 1950s to 1960 s - such as Hartree-Fock or the Kohn-Sham formulation of density functional theory (DFT) - enabled the birth of computational chemistry as a research field. Two decades later, computational catalysis began to flourish and increasingly became an established field with many significant contributions ${ }^{1}$.

The success of computational chemistry and catalysis goes hand-in-hand with the advances in method development and computing power. The early models consisting of just a few atoms have evolved into complex systems and interfaces. In heterogeneous catalysis, the materials gap is still preeminent, but significantly more realistic models are now routinely employed. Nanoparticles of over 1,500 atoms can now be treated at the DFT level ${ }^{2}$. Similarly, highthroughput studies are performed hosting a number of calculations that was simply unimaginable just a few years ago ${ }^{3}$. A major part of this success has been made possible by the use of supercomputing facilities. Since the first supercomputer was built in 1964, the computing performance of the top-tier machines has increased by eleven orders of magnitude ${ }^{4}$, contributing to the growth and consolidation of applied computational fields such as computational catalysis.

\section{The success of computational chemistry and catalysis goes hand-in-hand with the advances in method development and computing power. The early models consisting of just a few atoms have evolved into com- plex systems and interfaces.}

On 25 June 2018, the latest biannual release of the list of the 500 most powerful commercially available supercomputers was announced $^{4}$. This list, which now celebrates its twenty-fifth anniversary, ranks the computers based on their performance in a benchmark test of linear algebra equations measured in floating point operations per second (Flops). Summit, the brand-new supercomputer at Oak Ridge National Laboratory (USA), reached a maximum performance of 122,300 TFlops (1.223 X $10^{17}$ Flops) in the benchmark test, topping the list as the fastest supercomputer at the present time. When the Top500 list was first published back in June 1993, the CM-5 supercomputer, located at Los Alamos National Laboratory (USA) at the time, came top with a performance of 59.7 GFlops $\left(5.97 \times 10^{10}\right.$ Flops $)$, seven orders of magnitude below today's state of the art.

The US dominates the current list's top ten, featuring six of the computers, with the rest located in Asia (two in China and one in Japan) and Europe (Switzerland). The crown went back to the US after Chinese facilities had reigned for six years - the three initial years by the Tianhe-2 supercomputer, in Guangzhou, and the latter three by the Sunway TaihuLight machine, in Wuxi. Specifically, it returned to Oak Ridge, where the Titan computer, now ranked $\# 7$, once occupied the privileged spot in November 2012.

According to Moore's law, the performance of the top members of the list in the upcoming editions should reach the EFlops (ExaFlops, $10^{18}$ Flops) range in a couple of years. Based on each country's own plans and projections ${ }^{5,6}$, China is soon to be the first country to reach the exascale and are expected to by 2020 , with the Tianhe- 3 computer - although recent reports suggest a possible delay to $2021^{7}$. The US is expected to be the runner-up, with the Aurora-21 machine at Argonne National Laboratory expected by 2021. Japan and the EU would also join in 2022 onwards. However, the challenge this time is even higher, especially in light of the estimate of the energy consumption that will be required ${ }^{5}$.

Although the US and China together account for more than half of the current list's supercomputers, it is notable that 23 different countries from all continents appear in the top 250. Science is global, and the worldwide accessibility of highperformance computational resources ensures that excellence in research can be delivered in computational catalysis and other related fields.
Computational catalysis research has benefited from the increase in computing power in many different ways. More realistic models and more accurate methods are now being used, though some of these will also be considered outdated in the not too distant future. Catalytic reactions can now be explored on larger systems, namely supported nanoparticles ${ }^{8,9}$, solidliquid interfaces ${ }^{10}$ or explicit solution environments ${ }^{11}$ among others, using $\mathrm{ab}$ initio methods such as DFT. The improved accuracy is reflected in longer timescales in molecular dynamics ${ }^{12,13}$ or the implementation of high-precision approaches such as wavefunction-based methods ${ }^{14}$, global optimization algorithms ${ }^{15}$ or nuclear quantum effects ${ }^{16}$ on those aforementioned larger systems. It is worth stressing that simple results based on basic models can also be extremely valuable, and higher degrees of complexity should be introduced when they are required.

High-performance computing facilities are, and will continue to be, a fundamental pillar in the expansion of knowledge in catalysis. The development of new supercomputers with increased computing power will open the door to new and improved models and approaches. High-throughput strategies combined with artificial intelligence represent one such example in the foreseeable future ${ }^{17}$. We at Nature Catalysis are expectant about the continuing technological improvement and evolution of the field, and hope to host many of these exciting future discoveries.

Published online: 10 August 2018 https://doi.org/10.1038/s41929-018-0135-0

\footnotetext{
References

1. Thiel, W. Angew. Chem. Int. Ed. 53, 8605-8613 (2014).

2. Nanba, Y., Ishimoto, T. \& Koyama, M. J. Phys. Chem. C 121, 27445-27452 (2017).

3. Li, Q., Garcia-Muelas, R. \& Lopez, N. Nat. Commun. 9 526 (2018)

4. https://www.top500.org/

5. Bourzac, K. Nature 551, 554-556 (2017)

6. Service, R. F. Racing to match China's growing computer power, U.S. outlines design for exascale computer. Science Magazine (7 February 2018); https://go.nature.com/2LSIv4y

7. Giles, M. The US may have just pulled even with China in the race to build supercomputing's next big thing. Technology Review (11 July 2018); https://go.nature.com/2Lzd0Nz

8. Suchorski, Y. et al. Nat. Mater. 17, 519-522 (2018).
} 
9. Grønborg, S. S. et al. Nat. Commun. 9, 2211 (2018). 10. Stecher, T., Reuter, K. \& Oberhofer, H. Phys. Rev. Lett. 117 276001 (2016).

11. Mellmer, M. A. et al. Nat. Catal 1, 199-207 (2018).
12. Voitchovsky, K., Giofre, D., Segura, J. J., Stellacci, F. \& Ceriotti, M. Nat. Commun. 7, 13064 (2016).

13. Gaillac, R. et al. Nat. Mater. 16, 1149-1154 (2017).

14. Kubas, A. et al. J. Phys. Chem. Lett. 7, 4207-4212 (2016).
15. Kolsbjerg, E. L., Peterson, A. A. \& Hammer, B. Phys. Rev. B 97 195424 (2018).

16. Markland, T. E. \& Ceriotti, M. Nat. Rev. Chem 2, 109 (2018) 17. Tabor, D. P. et al. Nat. Rev. Mater 3, 5-20 (2018). 